\title{
Interface-rich Aqueous Systems for Sustainable Chemical Synthesis
}

\author{
Dainis Kaldre ${ }^{\mathrm{a}}$, Fabrice Gallou ${ }^{\mathrm{b}}$, Christof Sparr ${ }^{\mathrm{a}}$ and Michael Parmentier ${ }^{\mathrm{b} *}$
}

\begin{abstract}
Mimicking an enzyme's exquisite activity and selectivity is a long-standing goal for sustainable chemical method development in aqueous media. The use of interface-rich aqueous systems, such as single-chain polymers, micelles and vesicle membranes recently emerged as strategy to emulate the compartmentalization of natural systems. In aqueous solution, aggregates such as micelles or microemulsion droplets are formed, providing reaction environments different from bulk solutions that frequently improve selectivity and accelerate reaction rates for a wide array of chemical transformations. We present here selected examples of interface-rich aqueous systems and discuss the advantages they offer for chemical synthesis. In particular metal-catalyzed cross-coupling reactions are highlighted and future challenges to perform reactions in interface-rich aqueous media are discussed.
\end{abstract}

Keywords: Cross-coupling · Interface-rich aqueous systems · Sustainability · Synthesis · Water

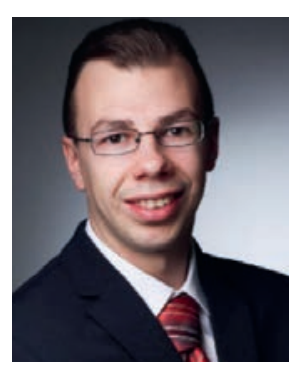

Dainis Kaldre was born in Riga (Latvia) and studied chemistry at the University of Latvia. In 2010, he moved to Canada to carry out his PhD studies at the McGill University in the group of Prof. James L. Gleason on development of new organocatalyzed transformations and asymmetric catalyst design. After graduation in 2016, Dainis pursued postdoctoral studies at University of Vienna with Prof. Nuno Maulide and with Prof. Christof Sparr at University of Basel. In 2019, Dainis joined F. Hoffmann La Roche in Basel.

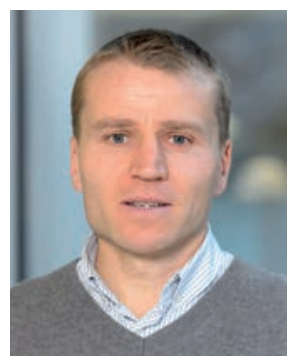

Fabrice Gallou received his $\mathrm{PhD}$ from The Ohio State University in 2001 in the field of natural products total synthesis. He then joined Chemical Development at Boehringer Ingelheim, USA, working as a process chemist responsible for route scouting and supply of early phase programs. He subsequently moved in 2006 to Chemical Development at Novartis, Switzerland, as a process development chemist, and became in 2008 responsible for global scientific activities, overseeing development and implementation of practical and economical chemical processes for large scale production of APIs. His research interests lie in the research and development of sustainable synthetic methodologies intended for large scale implementation.

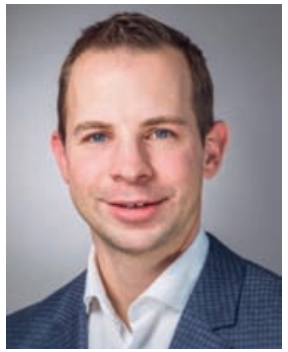

Christof Sparr carried out his $\mathrm{PhD}$ studies at the ETH Zurich working in the group of Prof. Ryan Gilmour and subsequently joined the groups of Prof. Dieter Seebach and Prof. Steven V. Ley as postdoctoral researcher. In 2013, Christof became habilitand mentored by Prof. Karl Gademann and since 2016, Christof is Assistant Professor of Organic Chemistry at the University of Basel. He is recipient of the ETH silver medal, a SNSF starting grant, the Werner Prize of the Swiss Chemical Society 2017 and the Ruzicka Prize of the ETH Zurich 2018.

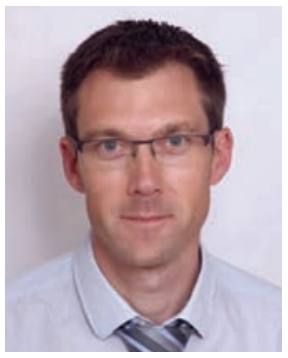

Michael Parmentier graduated from the University of Nancy (France) with a Masters degree in Chemistry. He moved in 2004 for his PhD-studies to the University of Montreal (Canada) to work with Prof. Hélène Lebel on the total synthesis of an interleukine inhibitor and on the development of a nitrene precursor for the aziridination reaction. In 2009, He moved back to Europe and carried out postdoctoral studies at the University of Basel under the supervision of Prof. Andreas Pfaltz where he gained insights into the organometallic field. During his stay, he closely collaborated with F. Hoffman La-Roche laboratories on the Hydrogen Isotope Exchange and with direct application on APIs. Since 2012 he is Process chemist in Novartis Pharma (Basel) in Chemical and Analytical development (CHAD). He is mainly involved in Early phase projects including transfer to pilot plant and in implementation of new technologies. Beside his scientific activities, he is coordinating exchanges with academia including setup of academic collaboration and leading internship programs. 


\section{From Enzymes to Interface-rich Aqueous Systems}

Enzymatic systems can accelerate chemical reactions with exquisite selectivity in aqueous media and under mild conditions. ${ }^{[1]}$ Promiscuous water-soluble enzymes frequently contain hydrophobic cavities that act as general bases or acids or accommodate metal ions to accelerate a wide array of chemical reactions. Structural simplifications of these characteristics by using soft interface-rich aqueous systems (IRAS), such as single-chain polymer nanoparticles, micelles, emulsion droplets and vesicle membranes have recently allowed to mimic several of the properties of enzymes, offering strategies for the sustainable organic transformation for a broad scope of substrates and reaction classes. In aqueous solution, these systems create environments different from bulk phase, allowing a high local concentration of reagents. Such interface-rich aqueous systems are distinguished into two types: ${ }^{[2]}$ type I systems that accelerate reactions by the local environment of the interface-rich structure and type II IRAS, which further promote chemical reactions with embedded catalytic sites.

\subsection{Single-Chain and Hyperbranched Polymers}

Single chain and hyperbranched water-soluble polymers in aqueous solution fold into globular structures, thus forming compartments with a specific local environment. The hydrophobic interior of such nanoparticles can be functionalized, for instance by immobilizing a chiral salen $\mathrm{Ti}^{\mathrm{IV}}$, thus providing type II systems (depicted in Fig. 1, $\mathrm{PN}_{68} \mathrm{IS}_{4}$ ). In aqueous solution, the formed nanoparticles have an average diameter of $12 \mathrm{~nm}$ and similar to enzymes, lead to an increased activity and exquisite selectivity for the sulfoxidation of arylsulfides, while the catalyst can be recovered and reused without significant loss of activity. ${ }^{[3]}$

\subsection{Reactions in Micelles}

Micelles are formed spontaneously in water by surfactants at high enough concentrations (CMC: critical micelle concentration; ca. $\left.10^{-3}-10^{-4} \mathrm{M}\right)$. In polar solvents such as water, the hydrophobic tails of the surfactant create a specific local environment at the center of the micelle, while the hydrophilic part is located at the outside surface, similar to the hydrophilic amino acid residues of globular proteins. Micelles thereby create an excellent compartment of type I, with high local concentrations of catalysts and reactants in the core that lead to a significant acceleration of chemical reactions, frequently combined with an increase in yield and selectivity. Furthermore, a covalently bound catalyst to the surfactant's hydrophobic side chain creates a type II system for specialized applications. These two general principles have been applied to accelerate a wide variety of chemical reactions including cross-couplings, ${ }^{[4]}$ in concert with biocatalytic reactions ${ }^{[5]}$ as well as organocatalytic ${ }^{[6]}$ and photocatalytic ${ }^{[7]}$ transformations. Moreover, Balskus and co-workers recently applied the commonly used surfactant TPGS-750-M to accelerate the flux of styrene produced in Escherichia coli, followed by sequential FeTPPClmediated cyclopropanation (Fig. 2). ${ }^{[8]}$ Rate acceleration for the styrene production step is believed to occur via association of the micelles with the bacterial membrane to increase membrane permeability. This work demonstrates that micellar media can also be used in synthetic biotechnology in combination with chemical reactions.

\subsection{Microemulsion Droplets}

Upon addition of immiscible organic co-solvents (5-20\%) to micelles in water, solvent-swollen oil-in-water microemulsion droplets are formed. As reactions in micelles may suffer from precipitation and low initial solubility of reagents, they often become unsuitable for large-scale synthesis. In contrast, microemulsion droplets are thermodynamically more stable and thus better suited for the scale-up of the chemical transformations. ${ }^{[9]}$ An example of this type of catalysis is the Suzuki-Miyaura reaction (Fig. 3), where the reaction under conventional micellar media conditions ( 2 wt $\%$ of surfactant TPGS-750-M or Kolliphor EL in water) led to low yields and partial deprotection of the Boc group, while the addition of $10 \%$ toluene co-solvent created a microemulsion, giving significantly higher yields and shorter reaction times for this air-tolerant coupling reaction. ${ }^{[10]}$

\subsection{Reactions in Vesicle Membranes}

Various amphiphilic molecules in aqueous media aggregate to bilayer vesicles with hydrophilic head groups pointing to both surfaces. Contrary to micelles, these vesicles thus enclose an aqueous volume, creating a different environment to accelerate chemical reactions. For example, Walde et. al..$^{[1]}$ developed a protocol for the multicomponent Passerini reaction to access $\alpha$-acetyloxy carboxamides (Fig. 4). With the surfactant DODAB, the product was obtained in 58\% with significantly higher reaction rates than in organic solvents or water in absence of amphiphile $(33 \%)$. The Passerini reaction between the long chain aldehyde and isocyanide is believed to occur within the vesicle membranes with the carboxylic acid in close proximity to the cationic amphiphile head group. Interestingly, the vesicle system can be reused several times without loss of activity. ${ }^{[11]}$

\section{Cross-coupling Reactions in Interface-rich Aqueous Systems}

Cross-coupling reactions are the most frequently utilized transformations in the pharmaceutical industry after amide bond formation. ${ }^{[12]}$ With the limited resources of transition metals and the environmental impact of solvent use, the development of novel protocols with high catalyst turnover and recyclable reaction medium remain a main industrial challenge. ${ }^{[13]}$ While most reported cross-coupling reactions involve high catalyst loadings unsuitable for large-scale applications, reactions in micellar media can be carried out with reduced amounts of catalyst and often allow to recycle the catalysts and the reaction media (Fig. 5).[14] Furthermore, extensive optimization studies revealed a significant influence of the structure of the surfactant and the micellar size for
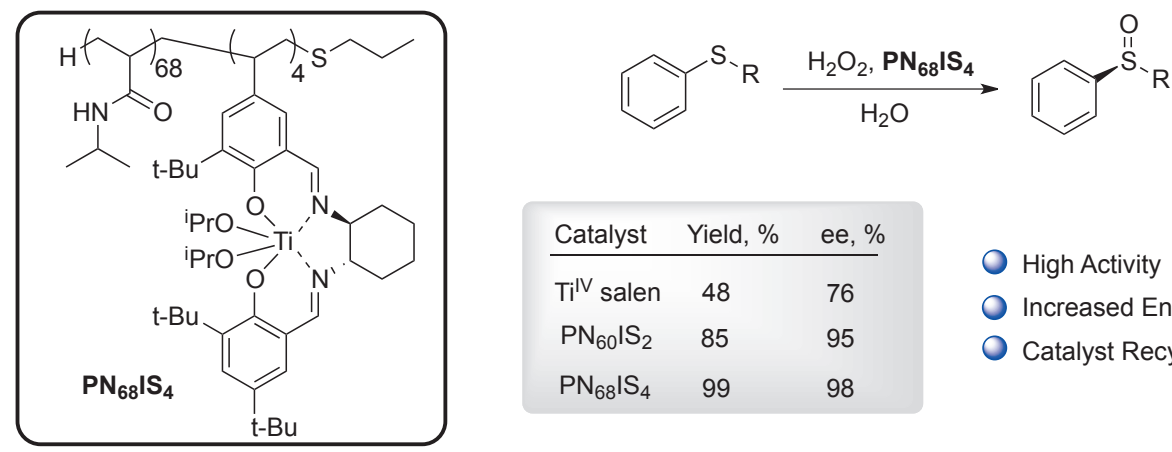

\begin{tabular}{lll} 
Catalyst & Yield, \% & ee, \% \\
\hline $\mathrm{Ti}^{\mathrm{IV}}$ salen & 48 & 76 \\
$\mathrm{PN}_{60} \mathrm{IS}_{2}$ & 85 & 95 \\
$\mathrm{PN}_{68} \mathrm{IS}_{4}$ & 99 & 98
\end{tabular}

High Activity
Increased Enantioselectivity
Catalyst Recycling

Fig. 1. Single-chain polymer with immobilized salen Tiv catalyst for the asymmetric sulfoxidation. 


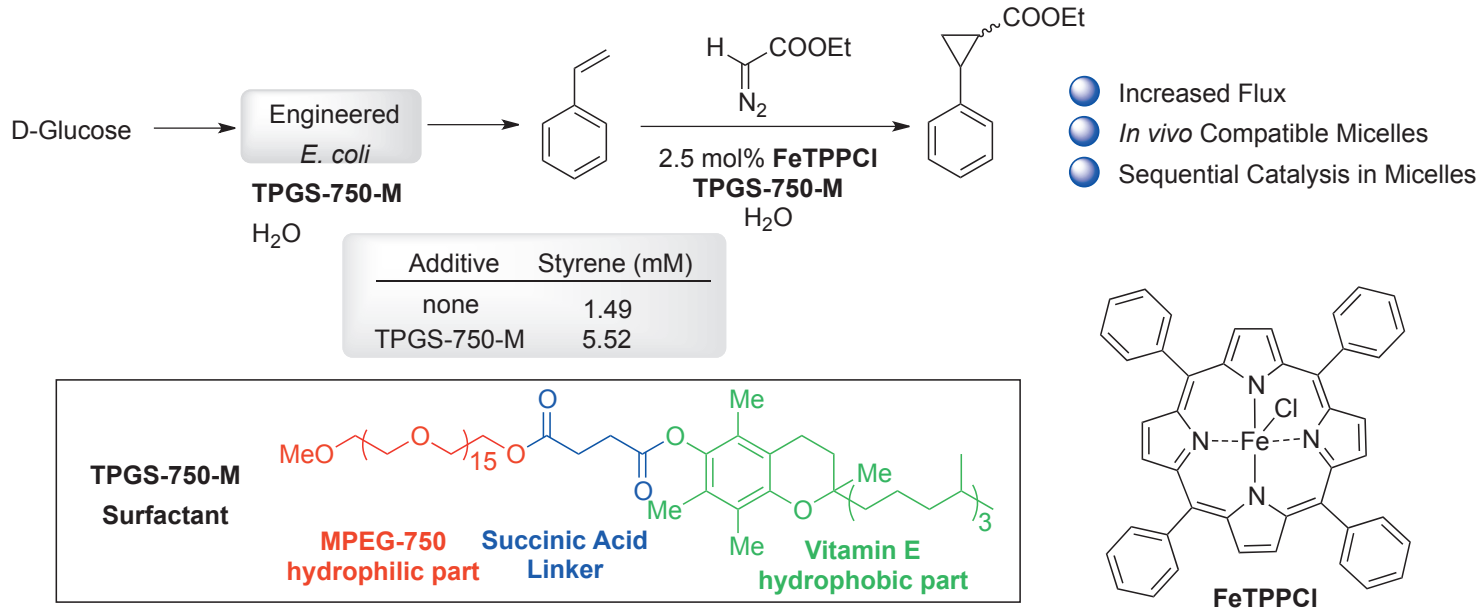

Fig. 2. Sequential styrene production in E. Coli followed by a cyclopropanation in micellar media.

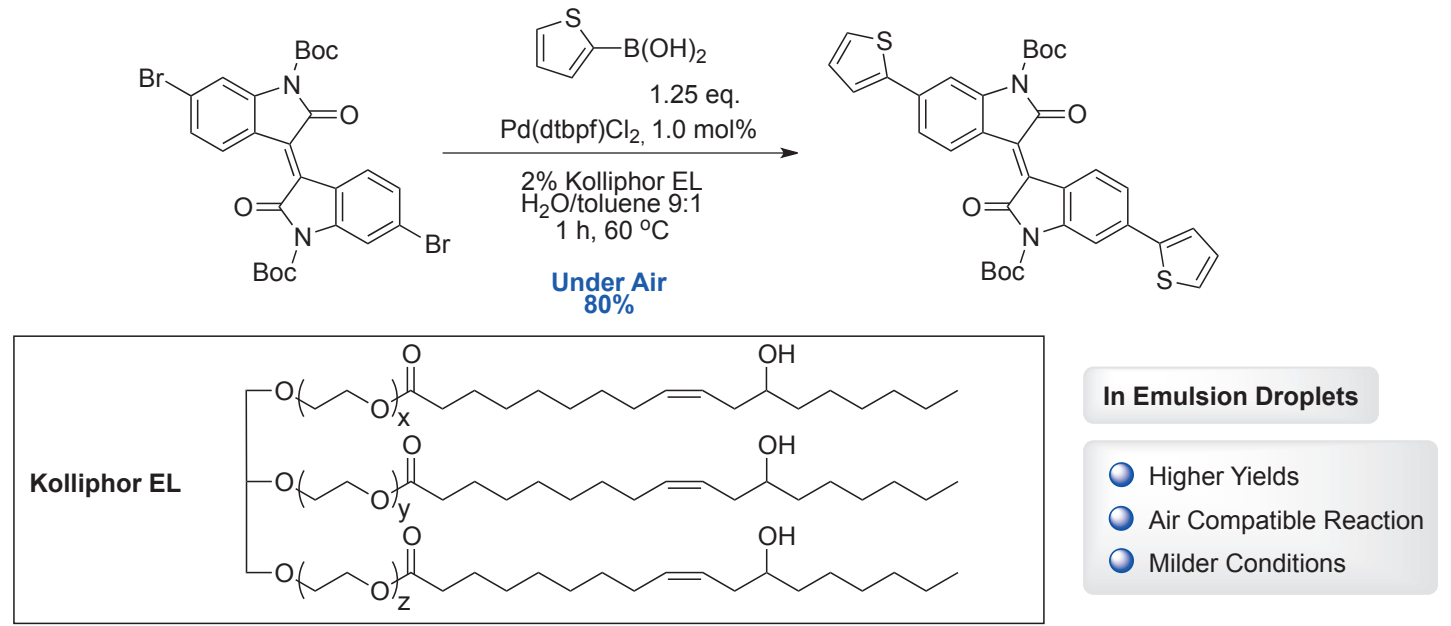

Fig. 3. Suzuki-Miyaura reaction in microemulsion droplets.

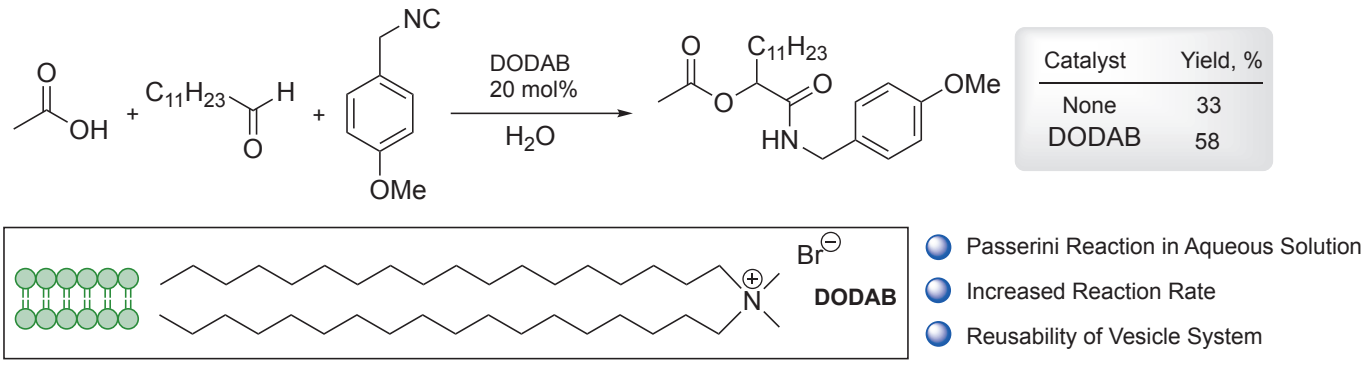

Fig. 4. Passerini reaction in vesicle membranes.

an optimal reaction outcome. ${ }^{[15,16]}$ Notably, the reactions in micellar media frequently depend on the reaction vessel, stirring speed and scale. However, high-throughput assay in microliter plates has been established to quickly optimize conditions for cross couplings in micellar media. ${ }^{[16]}$ Furthermore, the optimization of ligand electronic, steric properties and lipophilicity to favor longer residence time in the micelles allows for a high turnover frequency and prevents catalyst degradation in aqueous media. ${ }^{[17]}$

Remarkably, with Pd nanoparticles for the Suzuki-Miyaura coupling in micellar media at slightly elevated temperatures or at room temperature, the catalyst loading was recently reduced to as low as 320 ppm. ${ }^{[18]}$ To avoid degradation of Pd catalysts, Handa et. al. designed a new surfactant based on the proline scaffold (FI-750-M) that better mimics polar-aprotic solvents. This surfactant was effective in Suzuki-Miyaura couplings with polar quinolines ${ }^{[19]}$ and allows $\mathrm{sp}^{2}-\mathrm{sp}^{3}$ coupling reactions with air/mois- ture-sensitive $\pi$-allylpalladium intermediates. ${ }^{[20]}$ Moreover, other commonly utilized metal-catalyzed reactions such as BuchwaldHartwig amination, ${ }^{[21]} \mathrm{C}-\mathrm{H}$ activation, Heck reaction, ${ }^{[22]}$ Negishi coupling ${ }^{[23]}$ were performed at room temperature in aqueous media with the possibility to recycle the surfactant system.

Researchers at Novartis demonstrated that the production process of a new drug entity in a four-step sequence using the surfactant methodology improved the Process Mass Intensity (PMI) by $32 \%$, reduced overall time, improved the purity of the final product and significantly reduced the organic solvent PMI by $52 \%$ compared to the conventional route in organic solvents.[14] This study highlights that interface-rich aqueous systems offer not only more sustainable but also an economically feasible approach for process development. Interestingly, the highest cost reduction ( $38 \%$ vs. organic solvent process) was achieved in a metal-catalyzed cross-coupling step (Suzuki-Miyaura reaction, Fig. 6).[14] 

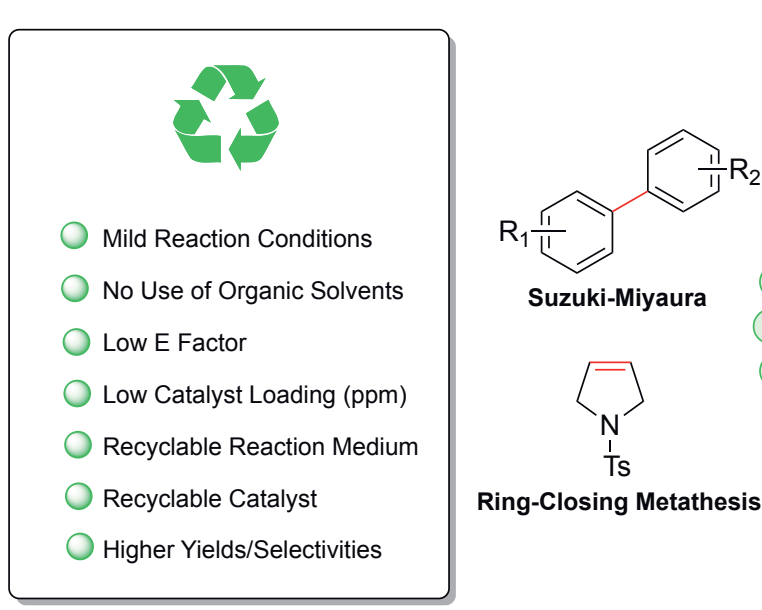

Ring-Closing Metathesis
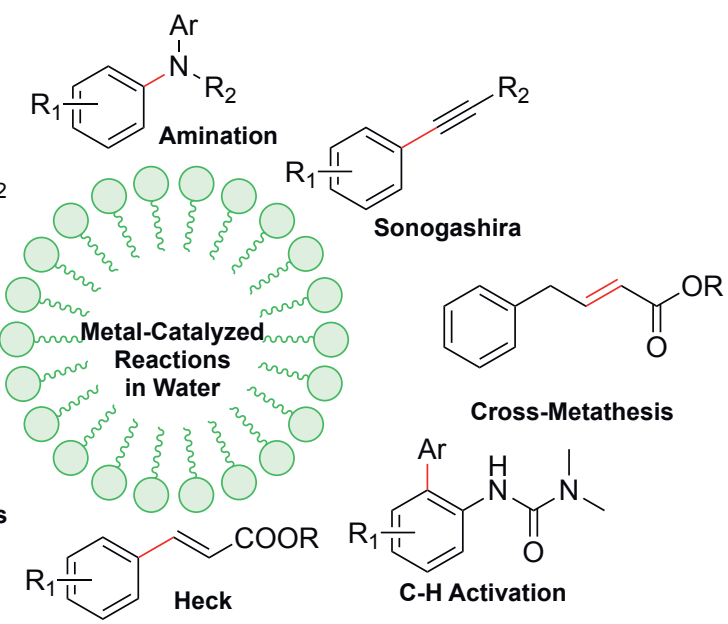

Fig. 5. Metal-catalyzed cross-coupling reactions in micellar media.

Similarly, the Heck reaction typically requires high temperatures and long reaction times in bulk. In 2008, Lipshutz et. al., applied micellar media to accelerate Heck reaction at room temperature (Fig. 6) to obtain high yields of up to $95 \%$.[24]

\section{Sonogashira Reactions in Micellar Media and Microemulsion Droplets}

The Sonogashira reaction is one of most frequently used crosscoupling reactions in academic research and the pharmaceutical industry to build propargylic derivatives. To make current protocols more sustainable, a combination of TPGS-750-M surfactant $(2 \mathrm{wt} \%)$ in water with Pd catalysts $(<3000 \mathrm{ppm})$ and different phosphine ligands (Fig. 7) was studied for a copper-free ${ }^{[25]}$ Sonogashira coupling under mild conditions $\left(25-45^{\circ} \mathrm{C}\right) .{ }^{[26]} \mathrm{ArI}$ and $\mathrm{ArBr}$ both were competent partners in this reaction, while ligand design was found to be crucial for the IRAS-accelerated reactions. As high concentrations can be reached in micelles, parameters such as ligand lipophilicity become particularly important for high turnover frequency.

To broaden the scope and applicability of aqueous Sonogashira reactions, a robust protocol with commercially available CataCXium A Pd G3 catalyst was recently developed in a particularly challenging cross-coupling (Fig. 8). ${ }^{[27]}$ With optimized reaction conditions using TPGS-750-M (2 wt\%) and organic cosolvent (i.e. 5\% THF), ${ }^{[9]}$ improved yields and selectivities were achieved for $o$-dihalogenated compounds compared to other catalyst/ligand combinations known for Sonogashira reactions.
Notably, the addition of glucose $(5 \mathrm{~mol} \%)$ further improved the catalytic efficiency and the homogeneity of the reaction mixture, rendering the protocol amenable for scale-up. ${ }^{[28]}$

The utility of the developed reaction system and the versatility of the micellar media was demonstrated in a three-step sequence to access an important chemical intermediate via a Sonogashira reaction followed by ester hydrolysis and finally an amide bond formation in micellar media (Fig. 9). This work demonstrates the possibility to apply micellar reaction media for several reactions in combination with metal-mediated cross couplings. ${ }^{[5,14,27]}$

An additional benefit of using micellar media in metal-catalyzed reaction is the efficient removal of residual metal that helps to comply with the strict health regulations. ${ }^{[29]}$ Simple isolation procedures, such as filtration of solid products or the extraction of products with environmentally benign solvents (e.g. EtOAc), typically amount to less than $1 \mathrm{ppm}$ residual $\mathrm{Pd}$ in the final products. ${ }^{[13]}$

\section{Conclusion and Outlook}

In the last decade, chemical synthesis in interface-rich aqueous systems evolved to a green and sustainable alternative to reactions in organic solvents. Many advantages including lower catalyst loadings, improved reaction rates and selectivities are already well documented. Future challenges lie in the detailed understanding of IRAS for the design of novel sustainable and robust transformations. The scalability was already proven on kilogram-scale, ${ }^{[14]}$ but has yet to be applied for a commercial route
Suzuki-Miyaura Reaction

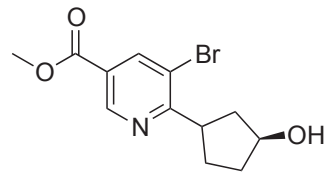

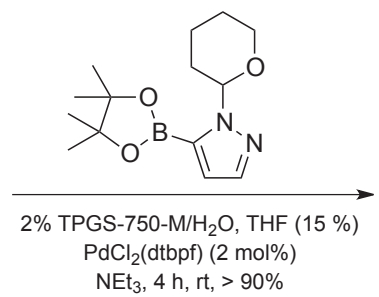<smiles>COC(=O)c1cnc(C2CC[C@H](O)C2)c(-c2ccnn2C2CCCCO2)c1</smiles><smiles>CCC(=O)/C=C/Cc1ccccc1OC</smiles>

Cross-Metathesis

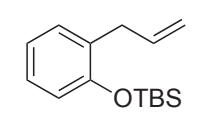

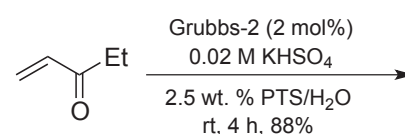

rt, $4 \mathrm{~h}, 88 \%$

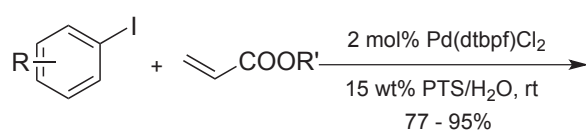<smiles>[R]OC(=O)C=Cc1cc#[R]cc1</smiles>

Fig. 6. Selected examples for metal-catalyzed cross-coupling reactions in micellar media. 


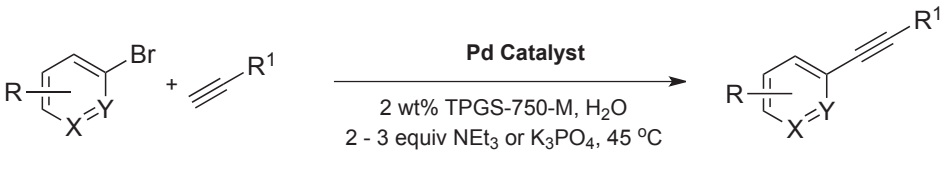<smiles>COc1cccc(OC)c1-c1cccc2c1P(C(C)(C)C)[C@@H](Cc1c([In]C)cc(C(C)C)cc1C(C)C)O2</smiles>

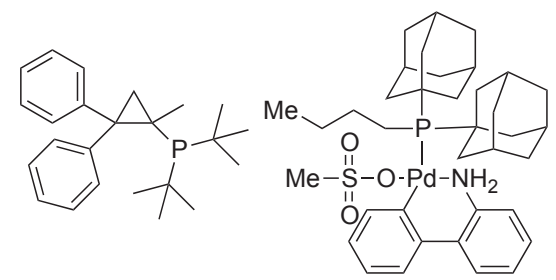

HandaPhos (1200 ppm) $\mathrm{Pd}(\mathrm{OAc})_{2}(1000 \mathrm{ppm})$

CBRIDP (3000 - 4500 ppm) $[\mathrm{Pd}($ cinnamly $)] \mathrm{Cl}_{2}(500-750 \mathrm{ppm})$
CataCXium A Pd G3 (3000 ppm)
Fig. 7. Sonogashira reaction in micellar media.

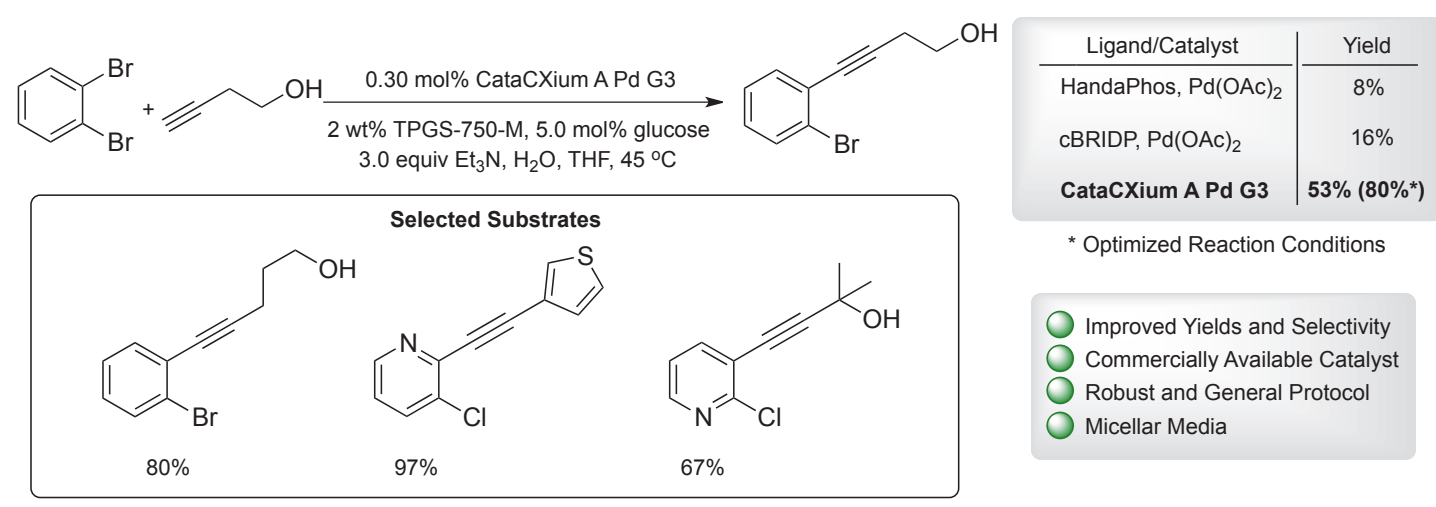

Fig. 8. Improved protocol for Sonogashira reactions in micellar media.

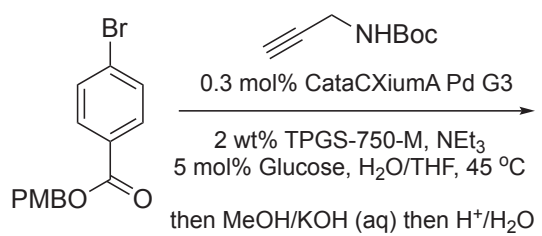<smiles>NCC#Cc1ccc(C(=O)O)cc1</smiles>

Fig. 9. Three-step sequence in micellar media.

in the chemical-pharmaceutical industry. In the future, we expect that the combination of IRAS with flow chemistry could render these processes continuous. More abundant metals (e.g. Fe, Ni) might be explored as alternatives to precious transition metals, while surfactants from renewable sources will become available on scale. Finally, catalysis in IRAS could enable novel chemical transformations, reaction networks, cascades or multi-component couplings. It will therefore be interesting to observe how catalysis in interface-rich aqueous media will expand for the years to come to further contribute to more sustainable chemical synthesis.

\section{Acknowledgements}

We gratefully acknowledge Novartis AG for financial support. C.S. gratefully thanks the Swiss National Science Foundation, (BSSGI0-155902/1 and 175746), the University of Basel and the NCCR Molecular Systems Engineering.

Received: June 27, 2019
[1] For selected directed evolution approaches and artificial metalloenzymes, see: a) F. H. Arnold, Acc. Chem. Res. 1998, 31, 125, DOI: 10.1021/ ar960017f; b) A. D. Liang, J. Serrano-Plana, R. L. Peterson, T. R. Ward, Acc. Chem. Res. 2019, 52, 585, DOI: 10.1021/acs.accounts.8b00618; c) C. Zeymer, D. Hilvert, Ann. Rev. Biochem. 2018, 87, 131, DOI: 10.1146/ annurev-biochem-062917-012034; d) S. N. Natoli, J. F. Hartwig, Accounts Chem. Res. 2019, 52, 326, DOI: 10.1021/acs.accounts.8b00586.

[2] S. Serrano-Luginbuhl, K. Ruiz-Mirazo, R. Ostaszewski, F. Gallou, P. Walde, Nat. Rev. Chem. 2018, 2, 306, DOI: 10.1038/s41570-018-0042-6.

[3] Y. Y. Zhang, R. Tan, M. Q. Gao, P. B. Hao, D. H. Yin, Green Chem. 2017, 19, 1182, DOI: 10.1039/c6gc02743a.

[4] Review articles: a) B. H. Lipshutz, S. Ghorai, Aldrichim. Acta 2012, 45, 3; b) G. La Sorella, G. Strukul, A. Scarso, Green Chem. 2015, 17, 644, DOI: 10.1039/c4gc01368a.

[5] M. C. Clerget, N. Akporji, J. Zhou, F. Gao, P. Guo, M. Parmentier, F. Gallou, J. Y. Berthon, B. H. Lipshutz, Nat. Comm. 2019, 10, 2169, DOI: 10.1038/ s41467-019-09751-4.

[6] B. H. Lipshutz, S. Ghorai, Org. Lett. 2012, 14, 422, DOI: 10.1021/ol203242r.

[7] L. Finck, J. Brats, B. Pavuluri, F. Gallou, S. Handa, J. Org. Chem. 2018, 83, 7366, DOI: 10.1021/acs.joc.7b03143.

[8] S. Wallace, E. P. Balskus, Angew. Chem. Int. Ed. 2016, 55, 6023, DOI: 10.1002/anie.201600966.

[9] a) F. Gallou, P. Guo, M. Parmentier, J. Zhou, Org. Process Res. Dev. 2016, 207, 1388, DOI: 10.1021/acs.oprd.6b00190; b) C. M. Gabriel, N. R. Lee, F. Bigorne, P. Klumphu, M. Parmentier, F. Gallou, B. H. Lipshutz, Org. Lett. 2017, 19, 194, DOI: 10.1021/acs.orglett.6b03468.

[10] M. Rooney, S. Mattiello, R. Stara,A. Sanzone,P. Brazzo, M. Sassi, L. Beverina, Dyes Pigments 2018, 149, 893, DOI: 10.1016/j.dyepig.2017.11.044. 
[11] D. Paprocki, D. Koszelewski, P. Walde, R. Ostaszewski, RSC Adv. 2015, 5 , 102828, DOI: $10.1039 / \mathrm{c} 5 \mathrm{ra} 22258 \mathrm{c}$

[12] D. G. Brow, J. Bostrom, J. Med. Chem. 2016, 59, 4443, DOI: 10.1021/acs. jmedchem.5b01409.

[13] M. C. Bryan, P. J. Dunn, D. Entwistle, F. Gallou, S. G. Koenig, J. D. Hayler, M. R. Hickey, S. Hughes, M. E. Kopach, G. Moine, P. Richardson, F. Roschangar, A. Steven, F. J. Weiberth, Green Chem. 2018, 20, 5082, DOI: $10.1039 / \mathrm{c} 8 \mathrm{gc} 01276 \mathrm{~h}$.

[14] a) F. Gallou, N. A. Isley, A. Ganic, U. Onken, M. Parmentier, Green Chem 2016, 18, 14, DOI: 10.1039/c5gc02371h; b) M. Parmentier, C. M. Gabriel, P. Guo, N. A. Isley, J. Zhou, F. Gallou, Curr. Opin. Green Sustain. Chem. 2017, 7, 13, DOI: 10.1016/j.cogsc.2017.06.004.

[15] B. H. Lipshutz, S. Ghorai, A. R. Abela, R. Moser, T. Nishikata, C. Duplais, A. Krasovskiy, R. D. Gaston, R. C. Gadwood, J. Org. Chem. 2011, 76, 4379 , DOI: 10.1021/jo101974u.

[16] C. E. Brocklehurst, F. Gallou, J. C. D. Hartwieg, M. Palmieri, D. Rufle, Org. Process Res. Dev. 2018, 22, 1453, DOI: 10.1021/acs.oprd.8b00200.

[17] M. Bihani, T. N. Ansari, J. D. Smith, S. Handa, Curr. Opin. Green Sustain. Chem. 2018, 11, 45, DOI: 10.1016/j.cogsc.2018.03.002.

[18] S. Handa, Y. Wang, F. Gallou, B. H. Lipshutz, Science 2015, 349, 1087, DOI: 10.1126/science.aac6936.

[19] S. Handa, F. Ibrahim, T. N. Ansari, F. Gallou, Chem. Cat. Chem. 2018, 10, 4229, DOI: $10.1002 /$ cctc. 201800958 .
[20] J. Brals, J. D. Smith, F. Ibrahim, F. Gallou, S. Handa, ACS Catal. 2017, 7, 7245, DOI: $10.1021 /$ acscatal.7b02653.

[21] C. Salome, P. Wagner, M. Bollenbach, F. Bihel, J. J. Bourguignon, M. Schmitt, Tetrahedron 2014, 70, 3413, DOI: 10.1016/j.tet.2014.03.083.

[22] B. H. Lipshutz, B. R. Taft, Org. Lett. 2008, 10, 1329, DOI: 10.1021/ ol702755g.

[23] A. Krasovskiy, C. Duplais, B. H. Lipshutz, Org. Lett. 2010, 12, 4742, DOI: 10.1021/ol101885t

[24] B. H. Lipshutz, B. R. Taft, Org. Lett. 2008, 10, 1329, DOI: 10.1021/ ol702755g.

[25] M. Gazvoda, M. Virant, B. Pinter, J. Kosmrlj, Nat. Commun. 2018, 9, 4814, DOI: ARTN 4814 10.1038/s41467-018-07081-5.

[26] S. Handa, J. D. Smith, Y. T. Zhang, B. S. Takale, F. Gallou, B. H. Lipshutz, Org. Lett. 2018, 20, 542, DOI: 10.1021/acs.orglett.7b03621.

[27] M. Jakobi, F. Gallou, C. Sparr, M. Parmentier, Helv. Chim. Acta 2019, 102, e1900024, DOI: 10.1002/hlca.201900024.

[28] J. E. Camp, J. J. Dunsford, E. P. Cannons, W. J. Restorick, A. Gadzhieva, M. W. Fay, R. J. Smith, ACS Sustain. Chem. Eng. 2014, 2, 500, DOI: 10.1021/ sc400410v.

[29] ICH (International Council for Harmonisation of Technical Requirements for Pharmaceuticals for Human Use), Guideline Q3. 